\title{
Requirements for Complex Life
}

\section{Berntsen R G and Grøn Ø G*}

Oslo and Akershus University College of Applied Sciences, Faculty of Technology Art and Design. PB 4 St. Olavs Plass, NO-0130 Oslo, Norway

*Corresponding author: Grøn $\varnothing$ G, Oslo and Akershus University College of Applied Sciences, Faculty of Technology Art and Design. PB 4 St. Olavs Plass, NO-0130 Oslo, Norway, Tel: +47 672386 87; E-mail: Oyvind.Gron@hioa.no

Rec date: Sep 24, 2015; Acc date: Sep 30, 2015; Pub date: Oct 03, 2015

Copyright: () 2015 Berntsen R G, et al. This is an open-access article distributed under the terms of the Creative Commons Attribution License, which permits unrestricted use, distribution, and reproduction in any medium, provided the original author and source are credited.

\section{Abstract \\ In this review we discuss the most important chemical, geophysical, climatic and astrophysical conditions that are considered vital for the development of complex life on a planet.}

Keywords: Complex life, Origin of life, Conditions for life

\section{Introduction}

We are here presenting a review of ideas, theories and observations that have made it possible to establish certain decisive criteria for the development of complex life on a planet. It should be noted, however, that in this respect we are close to the beginning in the establishment of a new area of research. We have already seen, however, that some qualitative guesses have been replaced by quantitative empirical data during the last ten years thanks to new observational equipment at the Earth and in space. For example it has recently been shown that "the physical properties of the planets in our Solar system appear to be very typical when compared with those of the observed exoplanets" [1]. And a recent statistical investigation [2] concludes that there is a least $92 \%$ chance that we are not the only civilization the Universe will ever have.

In order to assess what is necessary to have life on a planet we will use the Earth as a model and learning tool. After all, we believe the Earth is probably a typical terrestrial planet of its size and it is the only place we know of for now where life has developed. A statistical view on the known exoplanets, concludes that the Earth and the other planets in the solar system are typical. The two factors that seem to be special for the solar system are the lack of a super-Earth (a terrestrial planet between 1 and 10 times the mass of the Earth), and the orbital properties of the planets (orbits in the solar system are close to circular) and perhaps more conducive for life [1].

\section{Elements Important for the Evolution of Life}

The first stars were made already about two hundred million years after the Big Bang. Before that time there were mostly hydrogen and helium together with electromagnetic radiation and possibly dark matter and dark energy in the universe, with very small amounts of heavier elements.

The French physicist Jean Baptiste Perrin (1870-1942) proposed in 1919 that nuclear reactions can provide the source of energy in stars. He realized that the mass of a helium atom is less than the mass of 4 hydrogen atoms, and that the mass-energy equivalence of Einstein $\left(\mathrm{E}=\mathrm{mc}^{2}\right)$ implies that the combination of 4 hydrogen atoms into 1 helium atom would liberate enough energy to let stars produce radiation for billions of years. The British astronomer Sir Arthur
Eddington (1882-1944) followed up this idea in 1920. Based on precise measurements of atomic masses he argued that stars produced their energy from nuclear fusing of hydrogen into helium. He also suggested that heavier elements could be produced in stars. During the 1930's a more detailed understanding was developed by Bethe, von Weizsacker and other scientists. The theory of stellar nucleosynthesis was born.

In 1946 the British astronomer Sir Fred Hoyle (1915-2001) authored the first paper describing how heavier elements could be produced in stars. He later was part of a group of scientists who met in Cambridge. They systemized the ideas on how the heavier elements are produced and in 1957 they authored the famous B2FH paper (from the initials of the authors: Burbidge, Burbidge, Fowler, Hoyle) in which the field of nucleosynthesis was given a systematic treatment [3]. Part of this theory was published by Hoyle in 1954 with his supernova shell theory.

Eventually the stars are spreading much of their material into space at the end of the stellar life. Thus, we now know that almost all the elements needed for life are produced in the stars. Hence, literally we are the children of the stars.

Hydrogen and helium are still the most abundant elements in the universe. The estimated abundance of the ten most common elements in the Milky Way Galaxy is shown in Figure 1.

\begin{tabular}{|lc|}
\hline Hydrogen & $73,9 \%$ \\
Helium & $24,0 \%$ \\
Oxygen & $10400 \mathrm{ppm}$ (parts pr million, $10000 \mathrm{ppm}=1 \%)$ \\
Carbon & $4600 \mathrm{ppm}$ \\
Neon & $1340 \mathrm{ppm}$ \\
Iron & $1090 \mathrm{ppm}$ \\
Nitrogen & $960 \mathrm{ppm}$ \\
Silicon & $650 \mathrm{ppm}$ \\
Magnesium & $580 \mathrm{ppm}$ \\
Sulfur & $440 \mathrm{ppm}$ \\
\end{tabular}

Figure 1: Hydrogen and helium still amounts to $97.9 \%$ of the visual mass in the Milky Way. The ten most abundant elements combined are $99.9 \%$ of the visual mass: 
Page 2 of 9

But the Earth is different. While it is for example 7 times more carbon than silicon in the Milky Way there is about 220 times more silicon than carbon in the Earth. Silicon is a very important element for the development of life on the Earth, because it forms silicates which make up much of the land areas that are not as heavy as basalt. Therefore the silicon rich continents float upon the basalt beneath. All of this makes the Earth a terrestrial planet even though iron and oxygen makes up $31.9 \%$ and $29.7 \%$ of the Earth respectively. Most of the iron is in the central nucleus of the Earth.

It is a common assumption that life is based on the elements carbon, hydrogen and oxygen. But it has also been speculated whether life could be based on other elements, like silicon. Silicon has many similar chemical properties as those of carbon, and it is the eighth most abundant element in the Milky Way. However, there are more carboncompounds than the total number of compounds of the other elements with exception of hydrogen.

Water is important for life on Earth, and there are large amounts of water in the Universe. Among the most important properties of water are: The density of ice is smaller than that of flowing water. Hence in large lakes the cooling during the winter causes ice at the surface of the lakes, but there will remain flowing water beneath the ice. This permits life to go on during the winter in the lakes.

Pohorille and Pratt [4] have discussed which properties of water are most important for the evolution of life. They point out that a cell, or even a protocell, is a complex system whose structure and functions are largely modulated by non-covalent interactions. Indeed, noncovalent interactions-interactions that do not involve making or breaking chemical bonds-play a central role in almost all processes essential to terrestrial life. This is an advantage because it means that only a small energy is required to form molecules essential for life. Organic molecules can be dispersed in water and move freely around. Besides transmitting electrostatic interactions, water exhibits a remarkable trait that it also promotes hydrophobic interactions between nonpolar molecules or groups, rich in hydrocarbons.

Water has a large heat capacity, which is advantageous for stabilizing the climate on a planet with large oceans, since currents in the ocean tend to equalize the temperatures of hot and cold regions on a planet. Methane does not have such advantageous properties [4]. There don't seem to be other fluids-at least not in sufficient amounts-that are equally suitable for evolution of life.

Hydrogen is everywhere in space, and carbon and oxygen are the two most abundant elements produced in the stars. Oxygen and hydrogen form water, and carbon is the key element in a rich variety of organic molecules in the Milky Way. The basic idea is that only molecules formed around carbon in combination with water would probably be the basis for life because of the properties and the abundancy of these elements in the universe. For these reasons we will concentrate on water and carbon in discussing possible life on other planets.

\section{How did Life Begin on the Earth?}

Geological data indicate that life started on the Earth already between 3.8 and 3.5 billion years ago. This was just after the heavy bombardment when the Earth was hit by objects from the outer solar system. These bodies, asteroids and comets, probably delivered much of the water and other elements which finally became part of the Earth as we see it today. Such a cataclysmic period may be typical of new planets and may have contributed to the development of favorable conditions for the evolution of life.

Life appeared on the Earth in the form of simple microorganisms. We shall call this microbial life. The first era with only microbial life, lasted for about 2 billion years. 2.5 billion years ago, the cyanobacteria appeared. They were able to utilize the energy in the radiation from the Sun to synthesize carbohydrate molecules such as sugar from water and carbon dioxide, thus storing the energy from the Sun into chemical energy. In the process oxygen is liberated as a by-product. After the iron in the ocean and on land had oxidized, the atmosphere of the Earth got an increasing amount of free oxygen. The oxygen removed the methane which reduced the greenhouse effect. The Earth went into a 300 million year ice age, lasting from 2.4 to 2.1 billion years ago, (the Huronian glaciation). At that time there was less radiation from the Sun, and the methane and large amounts of carbon dioxide in the atmosphere was necessary to have liquid water which was important for the evolution of life. Melezhik has pointed out multiple causes for the Huronian glaciation [5]. A decrease of the $\mathrm{CO}_{2}$ level due to increased silicate weathering of fresh basaltic surfaces may have contributed to the change in surface temperature.

At the end of this ice age the evolution of life resumed, and multicellular organisms appeared about 1.5 billion years ago. Free oxygen meant that more complex forms of life, requiring a more effective metabolism, could function. The ozone layer formed when free oxygen flowed into the atmosphere. The ozone layer blocks UV radiation from penetrating the atmosphere and protects life on land and the upper part of the oceans.

The main point here is that the first primitive forms of life is necessary in order to modify the chemical properties of the environments, so that more complex forms of life could develop. On the Earth this process lasted for 2 billion years or more.

When NASA tries to discover signs of life on exoplanets they will look for such atmospheric properties. This is one of the main tasks for the James Webb Space Telescope (JWST) which NASA plans to put into space in October 2018.

A special case of complex life is intelligent life. We then think of beings taking a similar position on their planet as humans on the Earth, with technology, advanced communication and a high level of flexibility.

The first plants on land came only 500 million years ago, 3 billion years after life appeared on Earth. Vertebrates on land came 380 million years ago, and modern man is only 200 thousand years old. The era of the mammals, as one might say, started with the extinction of the dinosaurs 65 million years ago, due to a collision of an asteroid with the Earth.

In this article we shall discuss conditions that have been important for the evolution of complex life on the Earth, and use the knowledge obtained in this way to determine significant conditions for the evolution of complex life on other planets. There are obviously a lot of planets with conditions that are not suitable for the evolution of life. But there might also exist planets with conditions even better suited for the evolution of complex life that those on the Earth. It is important to take the time perspective into account when judging the possibility of life on a planet. One must expect that life on other planets needs a correspondingly long time for reaching a complex stadium such as that on the Earth. 


\section{What Makes a Planet Habitable?}

We need to distinguish between microbial life and multicellular, complex life. The key factor is the need for a more efficient metabolism in order for complex life to develop. An oxygen based metabolism can meet this requirement. In addition, complex life forms probably need an environment with more stability. For intelligent life with tools and technology to develop, we believe there must be land areas where these species can develop.

We will discuss some different parameters believed to be important for a planet to be habitable:

- A terrestrial planet with water and an atmosphere.

- Habitable zone around a star.

- The stability of the planet atmosphere.

- Plate tectonics, magnetic field and size of the planet.

- Orbital properties.

- A large moon.

- The size of the central star.

- Tidal effects of a small star.

- Habitable zone in a galaxy.

- The size of the galaxy.

- The duration of the period when a planet can support complex life.

\section{A Terrestrial Planet with Water and an Atmosphere}

The Earth was formed from the same disk of gas and dust as the rest of the solar system. The theory about how this happened is still under development. The discovery of other "solar" systems has shown a great variety. It is believed that the Earth together with the other terrestrial planets was formed inside the "snow line" in the solar system. Here the material was probably devoid of water and also carbon and nitrogen because these elements couldn't form solids in the hot nebular gas. Therefore water and other important materials for life presumable came to the Earth later in the process. Although details about the formation are still unclear, we know that the Earth went through a period of heavy bombardment hit by object from the outer solar system. These bodies, asteroids and comets, probably delivered much of the water and other elements which finally became part of the Earth as we see it today.

The primordial heat kept the interior of the Earth liquid, and probably plate tectonics started some time in this early period. Continents started to grow by the production of materials that could float on the heavier basalt below. Our Moon may have been created as the result of a collision between the early Earth and another planetsized object. The Earth ended up with its axis slightly tilted creating seasons, and the big Moon acted by stabilizing the tilt-angle. How rear is this combination of planet properties? We do not know, but perhaps we are on the brink of being able to observe some exoplanets in enough detail to get an idea about this crucial question.

\section{The Habitable Zone Around a Star}

The concept habitable zone has been in common use from around 1950. However, Whehell [6] mentioned such a zone already in 1853. He called it "The temperate zone of the solar system".

One may define the concept 'habitable zone' in the following way: A planet is in the habitable zone of a star if the radiation balance gives a surface temperature sufficiently high that there can be liquid water on the planet but not so high that the water vaporizes and disappears from the planet. The underlying conception is that life is based on compounds of carbon dispersed in water. Even if we do not exclude the possibility of other forms of life, this is the only form we know of.

The possibility that other stars than the Sun could have planets of suitable size in the habitable zone for a sufficiently long time that complex life could develop, and lead to a population of intelligent being with an advanced technology, lead to the establishment of SETI (Search for Extra Terrestrial Intelligence) in 1961. Then, in 1964 a systematic search for signals from external civilizations was started, but so far without definitive positive results after 50 years.

The space observatories gave us new knowledge about the solar system and the world outside. Especially we have obtained a very large amount of new and detailed information about the planets, moons and other bodies in the solar system. Mars has been a goal of particularly extensive investigations by several rovers moving on the surface of this planet. It has become clear that there has been a long period, lasting from 4 to 2 billion years ago, with extensive surface water. This has motivated to speculations whether there has been microbial life on this planet. So far no direct evidence of life has been found.

1956 planets in 1239 planetary systems including 488 multiple planetary systems, have been discovered as of 12 . September 2015. The Kepler space telescope has also detected a few thousand candidate planets. Statistics based upon these discoveries indicates that there are several billion planets in the habitable zones of their mother stars in the Milky Way galaxy.

Since the temperature on a planet depends upon the composite of the atmosphere due to the greenhouse effect, which changes with time, the changes in the greenhouse effect may lead the planet out of the habitable zone. Alternatively such changes may keep it there even when the zone is displaced due to changes in the mother star.

A planet may also receive frictional heat from tidal effects. Since the tidal effects decrease rapidly with distance, this will be most important for planets around small stars where the habitable zone is much closer to their mother stars than the Earth to our Sun.

In appendix A we have shown how the displacements of the habitable zone can be calculated. Radiation energy balance gives for the average surface temperature of the planet

$$
T=\left[\frac{(1-A) S}{2 \sigma(2-\varepsilon)}\right]^{1 / 4} \quad \text { (1)where } \sigma=5,67 \cdot 10^{-8} W / m^{2} K^{4} \text { is the }
$$

Stefan-Boltzmann constant, $\mathrm{A}$ is the planet's albedo (reflection coefficient), $\mathrm{S}$ the star constant corresponding to the Solar constant $\mathrm{S}=1,37 \mathrm{~kW} / \mathrm{m} 2$ at the Earth, i.e. $\mathrm{S}$ is the incoming radiation outside the atmosphere per unit surface area normal to the direction of the incoming radiation, and is the absorptivity of the planet's atmosphere. For the Earth it is usual to put $A=0,26$ and $\varepsilon=0,8$. The absorptivity of the atmosphere represents the greenhouse effect. With no greenhouse effect we have $\varepsilon=0$, and with maximal greenhouse effect where the atmosphere acts as a black body, we have $\varepsilon=1$. (Calculating the greenhouse effect is rather complicated so here we make use of a simplified formula which still demonstrates what we like to show.) By setting albedo $=0.3$ and no atmosphere, $\varepsilon=0$, we will get $\mathrm{T}=255 \mathrm{~K}(-18$ Celsius). Including a typical $\varepsilon=0.76$ will raise $\mathrm{T}$ to $287.4 \mathrm{~K}(+14.4 \mathrm{C})$ which is considered close to the average temperature of the Earth. 


\section{Effect of Changes of the Planet Atmosphere}

We would like to see how vulnerable the temperature and conditions on the Earth are for changes in greenhouse effect and albedo, keeping in mind that another planet probably would follow the same rules.

Using equation (1) we find that changing the absorptivity and albedo, respectively, to $\varepsilon=0.5$ and $\mathrm{A}=0.26$ will lower the average temperature to $278 \mathrm{~K}(+5 \mathrm{C})$. This is still above freezing, but we have to keep in mind that the arctic areas have a lower temperature than the average global temperature and might freeze. This is very close to the situation during the last glaciation period more than 12,000 years ago. On the Earth most of the land areas are situated in the Northern hemisphere. When the continents move towards the poles they provide a platform on which an ice sheet can form. Blocking of earlier ocean circulation can reduce moderation of the temperature between arctic and equatorial areas. Thus we can easily get a permanent ice sheet over large areas increasing the albedo significantly. Increasing the albedo to $\mathrm{A}=0.4$ causes the average temperature to decrease to $-9 \mathrm{C}$, which causes a glaciation. Comparing these figures with results from ice cores obtained in Antarctica, the average temperature during the last glaciation period was $8 \mathrm{C}$ lower than present, which corresponds to approximately $+6 \mathrm{C}$.

It should be noted that the continents have got their present positions just by accident. The reason for this is plate tectonics which moves the land areas and makes mountains in more or less accidental positions over time. Although the current position of the continents is a short period of time in the geological scale, it will still last for several million years, and can have a tremendous impact on the life for many species.

If we make a similar hypothetic change increasing the temperature by setting $\varepsilon=0,8$ and $A=0,2$ we get $T=300 \mathrm{~K}\left(27^{\circ} \mathrm{C}\right)$. This is clearly within a habitable temperature and should not make things dramatically difficult. Summing up we conclude that the Earth, for the time being, is much closer to the outer limit of the habitable zone than to the inner boundary. This might look surprising based on the common assumption that the Earth is close to the inner limit of the habitable zone. Hence, a habitable planet needs some leeway inside the theoretical habitable zone boundaries in order to allow for occasional changes in the atmosphere and planet orbit.

Based on the conclusion that the Earth for the time being is closer to the outer boundary of the habitable zone than the inner limit, we would like to point at some events in the early history of the Earth. The atmosphere is supposed to have contained great amounts of methane and carbon dioxide before the photosynthesis started. This explains that the Earth could have fluid water despite the fact that the luminosity of the Sun was much lower at that time. Methane has a strong greenhouse effect 72 times more than the same amount of carbon dioxide. After the "great oxygen event" 2.4 billion years ago, when cyanobacteria started photosynthesis which produced oxygen, the free oxygen in the atmosphere is believed to have removed the methane by converting it to carbon dioxide and water. Thus the greenhouse effect became smaller, and the temperature decreased. At the same time increased weathering reduced the amount of carbon dioxide. Consequently some areas froze, and the albedo increased. This led to the longest glaciation period we know of on the Earth-the Huronian ice age lasting for approximately 300 million years.

Much later, beginning 2.6 million years ago, the Earth went into the recent ice age with a number of glaciation periods. Each of the last glaciation periods have lasted close to 100000 years with much shorter mild periods in between. Plate tectonics is believed to have caused this situation. The Indian plate collided with Asia many million years ago lifting up the Himalaya plateau, so it is covered with snow all year long. This mountain used to be sea bottom before, and the material erodes rapidly, increasing the formation of limestone, which have taken carbon dioxide out from the atmosphere. A greater part of the continents of the Earth is now concentrated in the Northern hemisphere where an ice cap easily can form. These continents are blocking most of the ocean currents to the arctic, increasing the temperature differences on the planet.

Other planets harboring complex life must be expected to go through similar phases changing the composition of its atmosphere and position of land areas or elevation of mountain areas. Therefore there must be a margin in the position of a planet within the habitable zone preventing the planet from being frozen over forever or entering a detrimental run away temperature increase.

\section{Plate tectonics, Magnetic Field and Size of the Planet}

Alfred Wegener described what he called continental drift already in 1912. He gave further developments of his theory several times until he perished during an expedition on Greenland in 1930. The theory of continental drift was rejected by several researchers because it was difficult to explain the mechanism behind such continental movements. The theory was fully acknowledged first during the second half of the twentieth century. It is now called the theory of plate tectonics.

The crust of the Earth is divided in several plates moving on a liquid layer of basalt. The land areas consist to a large degree of granite in our time. This is not as heavy as basalt, so it is floating on the basalt like rafts. The material on the land areas are up to 4 billion years old. The seabed is renewed continuously, and most of it is less than 100 million years old. The oldest part of the seabed slides below the land areas and melts when it is pressed into the basalt beneath. In this way sediments in old seabed will be melted. Volcanos appear in such regions, transporting upwards some of the material that previously existed as old seabed. This provides a recirculation of $\mathrm{CO}_{2}$ and other important material for life.

This is of great significance both for the regulation of the Earth's surface temperature and for the plants and their photosynthesis. Recirculation of $\mathrm{CO}_{2}$ and other chemicals takes place through the socalled carbon-silicate cycle. In this planet-wide geochemical reaction, near surface weathering of calcium silicate $\left(\mathrm{CaSiO}_{3}\right)$ rocks leads to the formation of quartz-like minerals, that is, silicon dioxide $\left(\mathrm{SiO}_{2}\right)$. At the same time, carbon dioxide $\left(\mathrm{CO}_{2}\right.$, for example from the atmosphere) combines with the residual calcium atoms to form calcium carbonate (limestone, $\mathrm{CaCO}_{3}$ ). When subducted to deeper sediments, elevated pressures and temperatures reverse this reaction, ultimately leading to volcanic outgassing of $\mathrm{CO}_{2}[7,8]$.

As mentioned above complex life would come to an end with without $\mathrm{CO}_{2}$ because there would then be no photosynthesis. Due to the relationship between this fact and the significance of plate tectonics for maintaining a sufficiently high $\mathrm{CO}_{2}$-level for photosynthesis to occur, it became clear that continental drift is necessary for maintenance of complex life. Studies of the conditions for plate tectonics have indicated that a planet with several times the mass of the Earth will probably not have plate tectonics due to a too high viscosity in the basaltic layers. Also it is believed that for the seabed to be 
Page 5 of 9

subducted water is needed. Without recirculation of carbon, complex carbon based life is not possible.

The plate tectonics depends upon a liquid magma inside the Earth, which requires a sufficiently high temperature. Initially the temperature of the Earth was high and much of the Earth was liquid due to conversion of gravitational energy to thermal energy when the Earth was formed. Eventually much of the thermal energy was radiated out into space, the Earth cooled down, and the thickness of the external solid state crust increased.

Inside the Earth there is an additional heat source: radioactivity. Fission of the radioactive elements ${ }^{232}$ Thorium, ${ }^{238} \mathrm{Uran}$ and ${ }^{40}$ Potassium (with half-lives respectively $14.5,4.5$ and 1.25 billion years) provides energy preventing the solidification of the Earth's interior. ${ }^{235}$ Uran (half-life 704 million years) has contributed to this heating in the early stages but with a much shorter half-life ${ }^{235}$ Uran is now very low in abundance. In a planet much closer to the central (and smaller) star, heating due to tidal forces might add to or replace radioactive heating and thus maintain plate tectonic.

The magnetic field of the Earth is believed be produced by the motion in the outer core of liquid iron alloy. The magnetic field of the planet deflects the solar wind and cosmic rays. Without this protection our atmosphere could have been lost to space.

\section{Orbital Properties}

From observation of other planetary systems we can see that the orbit of the Earth is probably more circular than is usual for many exoplanets. Perhaps this is caused by the solar system having as many as 8 planets which moderate each other's orbits to become close to circular [9]. An orbit with low eccentricity is regarded favorable in keeping the temperature nearly constant over the year.

The axial tilt of the Earth is $23.5^{\circ}$. This causes seasons that are important for reducing the temperature difference between equatorial and arctic regions on the Earth. A large moon stabilizes the axial tilt.

The evolution of complex life requires billions of years. Hence, in order that a planet shall be habitable the orbit must be stable during several billion years.

\section{A large moon}

It has been speculated that a large moon stabilizes the tilt of the planet axis. Our large Moon has probably kept the tilt within small variations over time, but has previously been much closer to the Earth. The Moon gains energy from the spin of the Earth caused by tidal force. Thus the Moon has slowed down the much faster spin of the Earth billions of years ago. Measurements show that the distance between Moon and the Earth increases by $38 \mathrm{~mm}$ per year while the day on Earth increases by 15 microseconds per year. The tidal waves have created a tidal zone on the beaches where life could migrate from the ocean to the land. The tidal influence by the Moon is approximately twice that of the Sun, but has been much more powerful long ago.

\section{The size of the central star}

Our Sun is slowly increasing its luminosity. Hence the habitable zone moves outwards in the Solar system with a velocity given by equation (A13) in Appendix A. In our time its luminosity is approximately $30 \%$ higher than when it was formed. This is caused by the pressure balance inside the core of the Sun as it converts more and more of the hydrogen to helium. The luminosity of our Sun will continue to increase, and eventually the Earth becomes too hot to keep its water. A smaller central star develops more slowly, and may therefore allow for a more lasting, stable environment for a planet in the habitable zone. Heller and Armstrong have suggested that a more habitable planet than the Earth should be circulating a slightly smaller star than the Sun [7].

According to equation (A17) a star of 0.6 solar mass would have luminosity $0.6^{4}=0.13$ of our Sun. For a planet to receive the same amount of energy as the Earth, it must have an orbit of radius $0.36 \mathrm{AU}$ (54 million $\mathrm{km}$ ). Using equation (A19) it therefore follows that the habitable zone will be 0.36 of the width of the habitable zone around our Sun. An eccentricity of the same amount as the Earth will cause a 0.36 times the deviation of the Earth's orbit. So everything will be proportional for the new orbit. The year will be shorter and close to 102 Earth-days long for a 0.6 solar mass star and orbit radius of 54 million $\mathrm{km}$. A possible side effect is that shorter seasons will cause smaller changes in the temperature for a planet with a tilt like the Earth. Presumable a planet in this situation will be less vulnerable of going outside the habitable zone compared to the Earth.

\section{Tidal Effects of a Small Star and Spectral Differences}

A difference between our Sun and a smaller star would be that the tidal forces varying with the inverse cube of the distance, and there would be a difference in the spectral distribution of its radiation.

This means that the tidal force from a 0.6 mass star would introduce 13 times the tidal forces the Earth gets from our Sun or 4.3 times the tidal forces when our Moon and Sun are in line (spring tide). Then of course a more eccentric orbit would add to a stronger tidal effect. Jackson et al. points out that tidal influence tends to circularize the orbit of a planet [10] and hence reduce the effect over time. A possible positive outcome is tidal heating of the interior of the planet. In the Earth small amounts of radioactive material decays and make up for much of the heat loss through the crust, hence keeping the interior of the Earth liquid and keeping the plate tectonic running. Quite possible the amount of these radioactive elements could vary dependent on the nebula which a planetary system is developed from. Tidal heating might be another source of heating, doing the same thing around a smaller star and for a longer time.

However, a side effect of less stellar mass is a lower surface temperature of the star, since the temperature is proportional to $\mathrm{M}^{5 / 8}$ for main sequences star with a mass a little less than that of the Sun. This changes the spectral distribution in the direction of somewhat longer wavelengths, which may be less favorable for the photosynthesis. Smaller stars also have more violent flares which could be a hazard for life on land.

\section{Habitable Zone in a Galaxy}

Ward and Brownlee [11] pointed out that only certain parts of a galaxy are suitable for planets with life. Too near the center of a galaxy intense radiation and frequent collisions inhibits the evolution of complex life on the plants. They argue that frequent extermination would put the life back to the microbial stage, requiring a new start of the evolution. In extreme cases a planet could be sterilized by such incidences. On the other side, far from the center of a galaxy the metal content is too low to permit the formation of rocky planets. 
Page 6 of 9

Piran and Jiminez [12] have analyzed the conditions in galaxies with a focus upon the frequency of gamma ray bursts (GRBs), i.e. of extremely energetic flashes of radiation with duration from a second to more than 1000 seconds. The short GRBs, lasting for less than 2 seconds, come from colliding, compact objects such as black holes and neutron stars, and the long ones from explosions of massive stars. The long GRBs were much more common more than 5 billion years ago when large stars with 'life times' of only some million years were much more usual than at the present time.

If the Earth is hit by a GRB from an exploding star in our part of the Milky Way, there will be produced large amounts of NO and NO2 in the atmosphere. It disintegrates the ozone layer and leads to extermination due to ultraviolet radiation from the Sun. Piran and Jiminez [12] conclude that GRBs were so usual in the large galaxies more than 5 billion years ago, that they prevented the evolution of complex life. During the last 5 billion years great galaxies like the Milky Way have had regions where the GRBs have been sufficiently infrequent to permit the evolution of complex life.

Piran and Jiminez write that in order for GRBs not to be life threatening one has to live in a large, old galaxy like the Milky Way. The condition that specifies galactic habitability is that the halo mass lies in the range $10^{11.5}<M / M_{\mathrm{e}}<10^{12}$, so as to give rise to a large galaxy with a large outer region in which the GRB rate is low, but where there is a sufficient number of stars. Furthermore the halo must be young enough so that stars with favorable mass for hosting habitable planets have not left the main sequence. For $\mathrm{M}>0.7 \mathrm{M}_{\mathrm{e}}$ this corresponds to an age $<20$ Gyr.

Recently Piran and Jiminez [13] have also discussed how a cosmological constant, A, representing dark energy, modifies the danger from GRBs for the evolution of complex life. They found that a A-dominated universe favors the survival of life against GRBs. The reason is that the dark energy causes repulsive gravity and hence an accelerated expansion of the universe. This slows the growth of cosmic structures and increases the mean intergalactic separations, reducing the number of nearby satellite galaxies likely to host large exploding stars causing catastrophic GRBs. They also show that our existence as advanced biological mechanisms favors values of $\mathrm{A}$ around the present and disfavors $\mathrm{A} ; 0$.

In their book "Rare Earth: Why Complex Life is Uncommon in the Universe" Ward and Brownlee [14] discuss the so called "Rare Earth hypothesis". According to this hypothesis the development of complex life on the Earth has required a rare combination of astrophysical and geological circumstances, implying that complex life is an extremely unusual phenomenon in the universe. Heller and Armstrong [12] argue against this hypothesis because we may easily imagine planets that are even better suited for the development of complex life than the Earth (see below).

This type of disagreement indicates that we still have a lack of knowledge concerning planets with life. In particular there is a lack of statistical knowledge since we still have not discovered evidence of life outside the Earth.

In a recent work by Morrison and Gowanlock [15] an interesting statistical view has been presented. Although the probability of being hit by a supernova explosion is lowest in the region where our solar system is situated, the number of habitable planets closer to the galactic center increases many fold. By calculating a mean time between extinction events for a planet and summing the periods with opportunity time for development of complex life and intelligent life over all available planets, they found that despite a higher probability for a planet closer to the galactic center of having a hit it is still more opportunity time available and increasing towards the galactic center. Morrison and Gowanlock have done a simulation also for both past time and the future showing the same tendency.

\section{How Long can a Planet Support Complex Life?}

On the Earth life has been maintained for more than 3.5 billion years. Complex life has been around for approx. 500 million years. We do not know why it took so long before complex life forms developed. Oxygen in the atmosphere seems to be one important factor and the time before photosynthesis producing free oxygen started. Cyanobacteria evolved 2.5 billion years ago; at least one billion year after life arose. Then the Huronian glaciation lasting for 300 million years and 1.5 billion years ago the first multicellular organism showed up. Then another billion years before life went on land.

The different steps in the development of life on Earth could be just one example of a variation of time scales we could imagine from a number of planets. If we look towards the future of life on Earth it seems not so promising. The Sun develops, according to star theory, and the energy produced by fusion will grow steadily at a pace of $10 \%$ increase per 1.1 billion years. From this fact one can calculate that the $\mathrm{CO} 2$ level in the atmosphere will decrease due to the carbon-silicate cycle until the plants starve to death perhaps at a CO2 level of $10 \mathrm{ppm}$. Lovelock and Whitfield discussed this dramatic conclusion in Nature in 1982 [16]. Lovelock with several other coauthors and other scientists, discussed this problem over several years, and estimates for the development of our biosphere now point toward a time span of 500 to 700 million years before plants will vanish. Animals (and humans) are dependent of the plant life so consequently we will perish as well. For the Earth the conclusion is that complex life will just be here for approximately 1 billion years of a total planet life time of around 10 billion years.

One may also wonder how long plate tectonic will work. This is a difficult question to answer. Some estimates are just 500 million years more with plate tectonics. If plate tectonics stops the regulation of the temperature together with recycling of needed material for life and a number of other functions will stop. Plate tectonics ensures keeping the continents in place and growing. In a world without movements, weathering could tear down land in a few tens of millions of years leaving the Earth as a water world.

What can we humans do about these things? These days our societies are occupied by human population growth, environmental issues and global heating. This is on a very short time scale compared to the geological development of our planet.

Then looking further into the future we still live in an ice age. The next glaciation will hit us some day and it will for sure be much more dramatic than a global temperature increase of a few degrees. If reducing the combustion of fossil fuel can help us control global heating, burning more of it in a few thousand years if a glaciation shows up can be a solution, a tool to maintain a mild climate. Thus keeping enough coal and oil for times to come could be handy. Maybe people have developed other means of keeping the heat up when time comes.

On the much longer time scale we have the sun increasing its energy output and threatening all complex life on Earth. One of the ideas showing up frequently is adjusting the orbit of the Earth. Moving the 
Earth farther away from the sun could solve a possible overheating problem. Using a rather large comet passing the Earth at regular intervals could possible increase the energy of the Earth and move it outwards. It sounds like a risky project but these days we have research programs with the aim of changing the orbit of objects which could collide with Earth. Perhaps the same technology could be used in a bigger scale.

If we could control the daily cloud base, perhaps we could have rather dense clouds in the day time reflecting much of the sun's radiation, while we could have a clear sky through the night leaving the Earth to radiate freely into space in order to prevent long term heating. We also know of material having a negative greenhouse effect due to aerosols in the atmosphere. Of course these ideas are just examples of speculations, illustrating that new ways of thinking are always possible.

\section{The Manifold of Requirements in Order that a Planet shall be Habitable}

Whether a planet is habitable or not depends upon a surprisingly large number of astrophysical and geological factors. A suitable distance from the mother star is the foremost and obvious requirement for the possibility of complex life on a planet. The planet must exist for at least 4 billion years in the habitable zone of the mother star. This requires that the orbit of the planet must be stable for several billion years.

Water is a decisive factor. There may not be enough water at the position where the planet is formed. Then water must be transported from outer more water rich regions in the planetary system of the mother star. This seems to require the existence of an asteroid belt and possibly also a belt of comets in the planetary system. Still the transport and deposition of water on the Earth is difficult. The water has to survive the collision with the Earth and remain on the Earth.

Outgassing from a newly formed planet may form a too thick atmosphere to form favorable conditions for the evolution of complex life. Also in a young planetary system of a newly formed star warmer than the Sun there will be more harmful UV-radiation than in the solar system. The mass of the star and the evolution of its surface temperature determine the distance of the habitable zone from the star, but also the amount of UV-radiation hitting a planet in the habitable zone. Too much UV-radiation may cause a planet not to be habitable even in the habitable zone.

Hence we should generalize the concept 'habitable zone' to include a parameter characterizing the intensity of UV-radiation received by a planet in the zone. This parameter should be normalized to be equal to 1 when the 'UV star constant' of the UV-radiation is equal to that of the Sun at the Earth.

We are still lacking detailed knowledge about many relevant circumstances that decide whether a planet is habitable. Although dwarf stars recently have been popular in the search of habitable exoplanets, the habitable zone is closer to these stars than in the solar system. At such close distances there may be more dangerous flares with UV-radiation although the average UV-luminosity of dwarf stars is less than that of the Sun. There may also be a strong stellar wind at such distances, which may blow away some of the atmosphere of the planet.

Furthermore, tidal forces decrease faster with distance than the gravitational force. Hence it increases faster with decreasing distance. Therefore, planets at small distances from the mother star are acted upon by stronger tidal forces. This may cause tidal locked rotation for the star with one hot side facing the star and one cold side pointing outwards. There will then be a narrow zone between the hot and cold region that could be habitable.

A fine adjustment of several factors seems necessary in order for a planet to harbor development of complex life, for example the state of the protoplanetary disk when the star is formed, how great outgassing there is from the interior of the new formed planet, the intensity and energy distribution of the radiation from the star, how strong the stellar wind is, the existence of other large planets in the planetary system, a liquid planetary interior making the production of a sufficiently strong magnetic field possible to protect the atmosphere from being blown away.

The orbital parameters are also important for the evolution of complex life. The tilt of the rotational axis, its precession and the shape of the orbit must all be finely tuned.

In addition there are several geophysical factors that must be in place, such as continental drift, volcanism and the distribution of the land areas on the surface.

\section{Speculations}

Several research reports on the possibility of life on exoplanets contain speculations on points where we are lacking observational data and do not have sufficient knowledge. Speculations are important and a factor pushing the research forward. Often the consequences of speculations and new ideas may be tested by mathematical modeling and simulations. In anticipation of coming observations with for example the James Webb Observatory the researchers may be a step in front of the observational results trying to test out new possibilities.

\section{Appendix: Planetary Habitable Zone - Definition and Evolution}

It has been usual to define the habitable zone as the zone where there can be fluid water for billions of years on the surface of a planet, i.e. where the surface temperature of the planet is between $T_{1}=373 \mathrm{~K}$ and $\mathrm{T}_{2}=273 \mathrm{~K}$. This may, however, be an unrealistically large temperature interval for the development of complex life. If the average surface temperature of the Earth, which is $288 \mathrm{~K}$, decreases by for example 10 degrees, then the conditions may be as in the Huronian glaciation where most of the Earth was covered by ice. If the temperature of the surface is higher than about, there would also be difficulties for complex life, and the rate of loss of hydrogen would be dangerously high over a billion year time scale. Hence is seems more realistic to choose, say $T_{1}=373 \mathrm{~K}$ and $T_{1}=278 \mathrm{~K}$ for the zone permitting evolution of complex life.

Usually one talks about one planetary zone of a star, indicating that it depends upon the properties of the star only. This has been a useful simplification in order to obtain conceptual simplicity, but now we need a more encompassing and physically realistic definition.

We shall here consider how changing luminosity, L, of a star changes the position of its habitable zone, and how albedo, $\mathrm{A}$, and greenhouse effect $(G)$ affect a planet's position relative to the habitable zone of a planetary system. If we consider a planet moving along a circular path around a star, it will have a fixed distance from the star. The habitable zone will move radially when $L$ changes and the surface temperature of the planet will change due to changes of the albedo and the greenhouse 
effect. This may be described by introducing a fictitious radial displacement of the planet relatively to the habitable zone. If for example an increasing greenhouse effect causes a temperature increase the planet would "move" inwards in the radial zone.

However, the actual physical situation is that the planet has no radial motion. Hence it is the radial zone that gets an outwards displacement due to the increased greenhouse effect. This means that the habitable zone is not a property only of the star. It depends also upon the physical properties of a planet. In the Solar system, for example the Earth and Venus have different habitable zones due to the great difference of the greenhouse effect of these planets. The habitable zone of Venus is farther out in the Solar system than the habitable zone of the Earth.

For these reasons we suggest introducing the concept "planetary habitable zone" (PHZ), defined by taking into account the planet's albedo and greenhouse effect when calculating at what distance from the star the planet's surface temperature is $T_{1}$ (a maximal temperature compatible with evolution of complex life) and $T_{2}$ (a corresponding minimum temperature). Also an agreement should be obtained on realistic values of $T_{1}$ and $T_{2}$ to be used in the definition of the PHZ.

Radiation energy balance gives for the average surface temperature of the planet

$$
T=\left[\frac{(1-A) S}{2 \sigma(2-\varepsilon)}\right]^{1 / 4},(\mathrm{~A} 1)
$$

where $\sigma=5,67 \cdot 10^{-8} \mathrm{~W} \mathrm{~m}^{2} \mathrm{k}^{4}$ is the Stefan-Boltzmann constant, $\mathrm{A}$ is the planet's albedo, $S$ the star constant corresponding to the Solar constant $S=1,37 \mathrm{~kW} / \mathrm{m}^{2}$ at the Earth, i.e. $S$ is the incoming radiation outside the atmosphere per unit surface area normal to the direction of the incoming radiation, and is the absorptivity of the planet's atmosphere. For the Earth it is usual to put $A=0,26$ and $\varepsilon=0$. The absorptivity of the atmosphere represents the greenhouse effect. With no greenhouse effect we have $\varepsilon=0$, and with maximal greenhouse effect where the atmosphere acts as a black body, we have $\varepsilon=1$.

The relationship between the luminosity of a star and the "star constant", i.e. received radiation per unit area at a distance $r$ from the center of the star, is $\mathrm{S}=\mathrm{L} / 4 \pi \mathrm{r}^{2}$. Let $\mathrm{S}_{1}$ be the value of the star constant at the inner boundary of the PHZ and $S_{2}$ the value at the outer boundary. Then

$$
T_{1}=\left[\frac{(1-A) S_{1}}{2 \sigma(2-\varepsilon)}\right]^{1 / 4}, T_{2}=\left[\frac{(1-A) S_{2}}{2 \sigma(2-\varepsilon)}\right]^{1 / 4}
$$

Hence

$$
\frac{S_{1}}{S_{2}}=\frac{T_{1}^{4}}{T_{2}^{4}} \text {.(A3) }
$$

Let the width of the PHZ be $\Delta r$. Then

$$
S_{1}=\frac{L}{4 \pi r^{2}}, S_{2}=\frac{L}{4 \pi(r+\Delta r)^{2}} \text {. (A4) }
$$

This gives

$$
\frac{S_{1}}{S_{2}}=\left(1+\frac{\Delta r}{r}\right)_{2}^{2},(\mathrm{~A} 5)
$$

or

$$
\Delta r=r\left(\sqrt{\frac{S_{1}}{S_{2}}-1}\right) \cdot(\text { A6) }
$$

Inserting the relationship (A3) gives

$$
\Delta r=\left(\sqrt{\frac{T_{1}^{2}}{T_{2}^{2}}-1}\right) r .
$$

With $T_{1}=373 \mathrm{~K}$ and $T_{2}=273 \mathrm{~K}$ we get $\Delta r=0.87 \mathrm{r}$. On the other hand $T_{1}=343 \mathrm{~K}$ and $T_{2}=278 \mathrm{~K}$ gives $\Delta \mathrm{r}=0.52 \mathrm{r}$. The width of the $\mathrm{PHZ}$ is approximately half the distance of its inner boundary to the star.

Equation (A7) shows that the width of the PHZ is proportional to the distance of its inner boundary from the star. As the star gets warmer and the PHZ moves outwards, the PHZ will broaden.

We shall now find the velocity of PHZ due to a change of the luminosity of the star, and of the albedo and greenhouse effect of the planet. We shall represent the greenhouse effect by only one parameter, the absorptivity, $\varepsilon$, of its atmosphere. From

$$
r^{2}=\frac{L}{4 \pi S_{1}}, S_{1}=\frac{2 \sigma(2-\varepsilon)}{1-A} T_{0}^{4}(\mathrm{~A} 8)
$$

we get

$$
8 \pi \sigma T_{0}^{4} r^{2}=\frac{L(1-A)}{2-\varepsilon} \text {.(A9) }
$$

Inserting this into equation (A7) we find how the width of the PHZ depends upon the luminosity of the mother star and the albedo and greenhouse effect of the planet,

$$
\Delta r=K_{\otimes} \sqrt{\frac{L(1-A)}{L_{\odot}^{(2-\varepsilon)}}} \Delta r_{\otimes}, K_{\otimes}=\sqrt{\frac{2-\varepsilon_{\Theta}}{1-A_{\Theta}}},(\mathrm{A} 10)
$$

Where $L_{\mathrm{e}}$ is the luminosity of the Sun, and $\varepsilon_{\otimes}$ and $A_{\otimes}$ are the absorptivity of the Earth's atmosphere and the albedo of the Earth, respectively. The formula shows that decreasing luminosity, increasing albedo and decreasing greenhouse effect (decreasing value of $\varepsilon$ ) makes the PHZ thinner, which decreases the probability of finding a planet within the PHZ.

Differentiating equation (A9) we find

$$
\frac{d r}{d t}=\frac{r}{2}\left(\frac{1}{L} \frac{d L}{d t}-\frac{1}{1-A} \frac{d A}{d t}+\frac{1}{2-\varepsilon} \frac{d \varepsilon}{d t}\right) .(\mathrm{A} 11)
$$

The physical interpretation of this equation is that the PHZ has a velocity

$$
v_{P H Z}=v_{L}+v_{A}+v_{G},(\mathrm{~A} 12)
$$

where

$$
v_{L}=\frac{r}{2 L} \frac{d L}{d t}(\mathrm{~A} 13)
$$

is the contribution to the velocity of the PHZ due to a changing luminosity of the star,

$$
v_{A}=-\frac{r}{2(1-A)} \frac{d A}{d t}(\mathrm{~A} 14)
$$

is the contribution due to a changing albedo, and

$$
v_{G}=\frac{r}{2(2-\varepsilon)} \frac{d \varepsilon}{d t}(\mathrm{~A} 15)
$$

is contribution due to a changing greenhouse effect. 
Page 9 of 9

The time taken for the PHZ to move so that a planet without radial motion changes its position relative to it from the outer boundary to the inner boundary is $\Delta t=\Delta r / V_{\mathrm{PHZ}}$. It follows from equations (A7) and (A11) that

$$
\Delta t=\frac{2\left(T_{1}^{2}-T_{2}^{2}\right)}{T_{2}^{2}\left(\frac{1}{L} \frac{d L}{d t}-\frac{1}{1-A} \frac{d A}{d t}+\frac{1}{2-\varepsilon} \frac{d \varepsilon}{d t}\right)} .(\mathrm{A} 16)
$$

This is a characteristic time telling how long a planet with circular path can remain inside the $\mathrm{PHZ}$ when the luminosity of the mother star and the albedo and greenhouse effect of the planet change. The characteristic time due to the change of the mother star's luminosity, only, is

$$
\Delta t_{L}=\frac{2\left[\left(T_{1} / T_{2}\right)^{2}-1\right]}{(1 / L)(d L / d t)} .
$$

The rate of change of the luminosity of a main sequence star is with good accuracy given by [16]

$$
L=\frac{5 L_{e}}{7-2\left(t / t_{e}\right)},(\mathrm{A} 18)
$$

where $L_{\mathrm{e}}=3,85 \cdot 10^{26} \mathrm{~W}$ is the present day solar luminosity, and $t_{\mathrm{e}}=4.47 \mathrm{Gyr}$ is the age of the Sun. Differentiating and inserting the present rate of change into equation (A17) gives

$$
\Delta t_{L}=\left[\left(T_{1} / T_{2}\right)^{2}-1\right] 5 t_{e} \text {. (A19) }
$$

Inserting $T_{1}=333 \mathrm{~K}$ and $T_{2}=278 \mathrm{~K}$ gives $\Delta t_{\mathrm{L}}=2.17 t_{\mathrm{e}}=9.8 \mathrm{Gyr}$. The characteristic times associated with changes of the greenhouse effect and the albedo are much shorter.

Furthermore equations (A10) and (A18) give the time evolution of the width of the habitable zone in the Solar system as

$$
\Delta r_{S}=\sqrt{\frac{5}{7-2\left(t / t_{e}\right)}} \Delta r_{e},(\mathrm{~A} 20)
$$

where $\Delta r_{\mathrm{e}}$ is the present width of the habitable zone in the Solar system.

Small stars emit less radiation than large stars. The luminosity of main sequence stars with mass $0.43 M_{\mathrm{e}}<M<2 M_{\mathrm{e}}$ is

$$
L=\left(\frac{M}{M_{e}}\right)^{4} L_{e}(\mathrm{~A} 21)
$$

where $M_{\mathrm{e}}$ is the mass of the Sun. Inserting this into equation (A9) we find that the distance of the inner boundary of the habitable zone has a distance from the center of the star,

$$
r=\left(\frac{M}{M_{\odot}}\right)^{2} r_{\odot},(\mathrm{A} 22)
$$

where $r_{\mathrm{e}}$ is the corresponding distance in the Solar system, i.e. $r_{\mathrm{e}}=1 \mathrm{AU}$. Inserting equation (A18) into equation (A7) we find how the extension of the habitable zone depends upon the mass of the mother star in this mass interval,

$$
\Delta r=\left(\frac{M}{M_{e}}\right)^{2} \Delta r_{e^{.}}(\mathrm{A} 23)
$$

For a star with $M=0.6 M_{\mathrm{e}}$, for example, this gives $\Delta r=0.36 \Delta r_{\mathrm{e}}$ or about the third of the width of the habitable zone in the Solar system. Hence the probability that a planet moving around such a small star is positioned within the habitable zone of the star is less than that of a planet in the Solar system. It may be noted that by inserting (A17) into equation (A10) the width of the PHZ is given as

$$
\Delta r=K_{\otimes}\left(\frac{M}{M_{e}}\right)^{2} \sqrt{\frac{1-A}{2-\varepsilon}} \Delta r_{\otimes}(\mathrm{A} 24)
$$

\section{References}

1. Martin RG, Livio M (2015) The Solar System as an Exoplanetary System. The Astrophysical Journal 810: 2.

2. Behroozi PS, Peeples M (2015) On the History and Future of Cosmic Planet Formation. Cornell University Library.

3. Burbidge E, Burbidge GR, Fowler WA, Hoyle F (1957) Synthesis of elements in stars. Rev Mod Phys 29: 547-650.

4. Pohorille A and Pratt AL (2012) Is Water the Universal Solvent for Life? Springer Science 42: 405-9

5. Melezhik VA (2006) Multiple causes of Earth's earliest global glaciation. Terra Nova, 18, 130-137.

6. Whewell W (1853) On the Plurality of Worlds. (1stedn), J. W. Parker and Son, London.

7. Heller R and Armstrong J (2014) Superhabitable Worlds. Astrobiology 14: 50-66.

8. Lacis AA, Schmidt GA, Rind D, Ruedy RA (2010) Atmospheric CO2: Principal control knob governing Earth's temperature. Science 330: 356-359.

9. Brian Jackson, Rory Barnes, Richard Greenberg (2008) Tidal heating of terrestrial extrasolar planets and implications for their habitability. MNRAS 391: 237-245.

10. Morrison IS, Gowanlock MG (2015) Extending Galactic Habitable Zone Modeling to Include the Emergence of Intelligent Life. Astrobiology 15: 8.

11. Ward P, Brownlee D (2002) The Life and Death of Planet Earth: How the New Science of Astrobiology Charts the Ultimate Fate of the World. Piatkus.

12. Piran T, Jimenez R (2014) On the role of GRBs on life extinction in the Universe. Phys Rev Lett 113: 231102.

13. Piran T and Jimenez R (2014) Cosmic explosions, life in the Universe and the Cosmological Constant. ArXiv: 1508.01034.

14. Ward P and Brownlee D (2000) Rare Earth: Why Complex Life is Uncommon in the Universe. Piatkus.

15. Lovelock JE, Whitfield M (1982) Life Span of the Biosphere. Nature 296: 561-563.

16. G. Feulner (2012) The faint young Sun problem. Rev Geophys 50. 